\title{
Author Correction: ZSCAN10 expression corrects the genomic instability of iPSCs from aged donors
}

Maria Skamagki, Cristina Correia, Percy Yeung, Timour Baslan, Samuel Beck, Cheng Zhang, Christian A. Ross, Lam Dang, Zhong Liu, Simona Giunta, Tzu-Pei Chang, Joye Wang, Aparna Ananthanarayanan, Martina Bohndorf, Benedikt Bosbach, James Adjaye, Hironori Funabiki, Jonghwan Kim, Scott Lowe, James J. Collins, Chi-Wei Lu, Hu Li, Rui Zhao and Kitai Kim

Correction to: Nature Cell Biology https://doi.org/10.1038/ncb3598, published online 28 August 2017.

In the version of this Article originally published, Supplementary Fig. 6j showed incorrect values for the LS and AG4 glutathione samples, and Fig. $5 \mathrm{c}$ and Supplementary Fig. $6 \mathrm{j}$ did not include all $n=6$ samples for the hESC, Y-hiPSC and AG4-ZSCAN10 groups as was stated in the legend. In addition, the bars for hESC, Y-hiPSC, AG4-ZCNAN10, AG4 and LS in Supplementary Fig. 6i and $\mathrm{j}$ have been reproduced from Fig. $5 \mathrm{~b}$ and c, respectively. Figure 6e was also reproduced in the lower panel of Supplementary Fig. 6h, to enable direct comparison of the data, however this was not explained in the original figure legends. The correct versions of these figures and their legends are shown below, and Supplementary Table 5 has been updated with the source data for all numerical data in the manuscript.
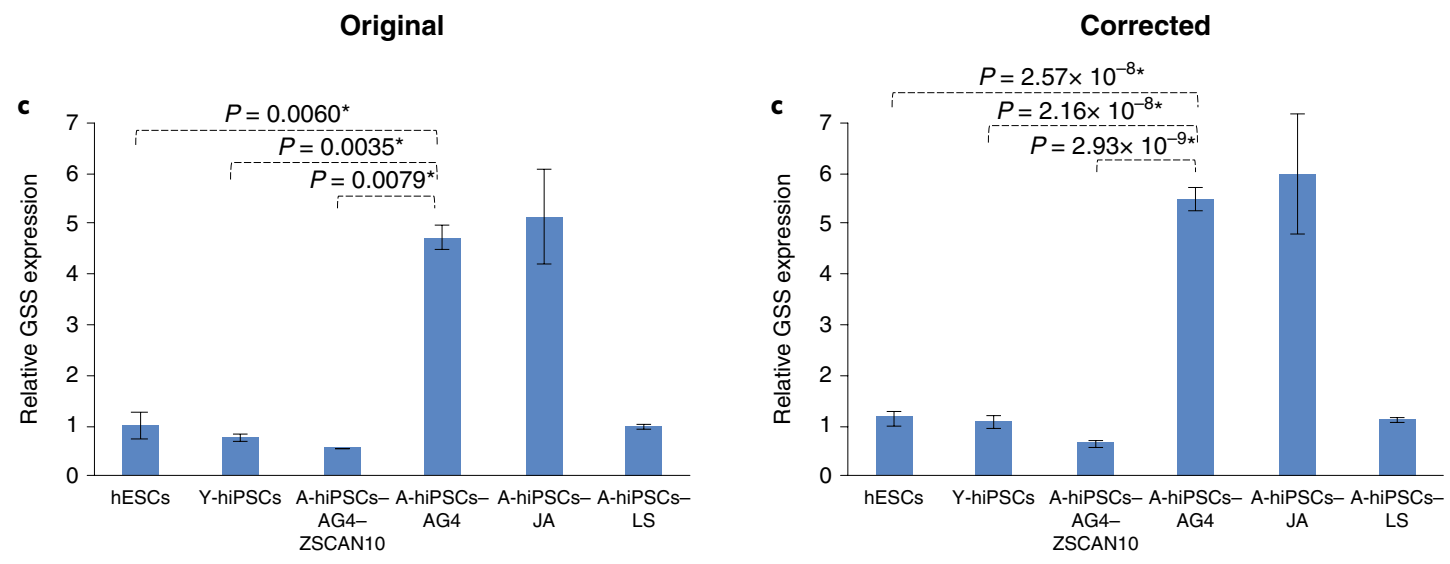

Fig. 5 | Evaluation of ZSCAN10 function in DNA damage response and genomic integrity in human A-hiPSCs. a, Immunoblots showing the levels of PATM and $\beta$-actin proteins with three imported A-hiPSC clones with known abnormal cytogenetic signature. b, qPCR of ZSCAN10. The error bars indicate s.e.m. of two replicates with three independent clones $(n=6)$ in each sample group, except three biological replicates $(n=3)$ in the sample of A-iPSC-JA and A-iPSC-LS. Statistical significance was determined by two-sided $t$-test followed by post hoc Holm-Bonferroni correction for a significance level of 0.05 ; ${ }^{*}$ indicates significant. Note that these data are also presented in Supplementary Fig. 6 i. c, aPCR of GSS. Statistical significance was determined by two-sided $t$-test followed by post hoc Holm-Bonferroni correction for a significance level of 0.05; *indicates significant. The error bars indicate s.e.m. of two replicates with three independent clones $(n=6)$ in each sample group, except three biological replicates $(n=$ 3 ) in the sample of A-iPSC-JA and A-iPSC-LS. (Note that these data are also presented in Supplementary Fig. 6j.) d, Immunoblots showing the levels of pATM and $\beta$-actin in five independent clones of A-hiPSCs, five independent clones of Y-hiPSCs and five clones of A-hiPSCs expressing ZSCAN10. e, Immunoblot showing impaired ATM DNA damage response in Y-hiPSCs with ZSCAN10 shRNA expression in three independent clones after phleomycin treatment $\left(2 \mathrm{~h}, 30 \mu \mathrm{g} \mathrm{ml}^{-1}\right)$. f, Copy number profiling analysis of Y-hiPSCs with ZSCAN10 shRNA expression in four independent clones ${ }^{43}$. Unprocessed original scans of blots are shown in Supplementary Fig. 7.

\section{Corrected Fig. 6 legend:}

Impaired DNA damage response in human A-hiPSCs caused by deregulation of ZSCAN10 and GSS and recovered by ZSCAN10 expression. a, Excessive oxidation capacity with elevated glutathione in A-hiPSCs, and recovery by ZSCAN10 expression. The total glutathione level was measured to determine the maximum oxidation capacity. Excessive oxidation capacity of glutathione in A-hiPSCs is normalized to the level of hESCs and Y- hiPSCs by transient expression of ZSCAN10. Glutathione analysis was conducted with the glutathione fluorometric assay. Mean \pm s.d. is plotted for three biological replicates with two independent clones $(n=6)$ in each sample group from each condition. Statistical significance was determined by two-sided $t$-test. b, ROS scavenging activity of hESCs, Y-hiPSCs, A-hiPSCs and A-hiPSCs-ZSCAN10. A cellular ROS assay kit (DCFDA assay) was used to measure $\mathrm{H}_{2} \mathrm{O}_{2}$ scavenging activity. A-hiPSCs show strong $\mathrm{H}_{2} \mathrm{O}_{2}$ scavenging activity, with a reduced response against treatment with TBHP (tert-butyl hydrogen peroxide; stable chemical form of $\mathrm{H}_{2} \mathrm{O}_{2}, 3 \mathrm{~h}$ ); the response is recovered by ZSCAN10 expression. Mean \pm s.d. is plotted for four biological replicates in each sample group from each condition $(n=4)$. Statistical significance was determined by two-sided $t$-test. c, Immunoblot of pATM showing recovery of 
the DNA damage response after phleomycin treatment in three independent clones of A-hiPSCs with shRNA-mediated knockdown of GSS. d, Immunoblot of pATM showing that lentiviral expression of GSS cDNA impairs the DNA damage response in three independent clones of Y-hiPSCs after phleomycin treatment. e-g, Copy-number profiling analysis of human iPSCs ${ }^{43}$. Schematic diagrams represent seven rearranged A-hiPSCs, four non-rearranged A-hiPSCs and five non-rearranged A-hiPSCs- ZSCAN10 in the genetically controlled setting of A-hiPSCs. Ten non-rearranged Y-hiPSCs, which were generated from a different tissue donor, were also included. A-hiPSCs ( $n$ $=11(7 / 11), P=0.64)$, (These data are also presented in Supplementary Fig. $6 \mathrm{~h})$, A-hiPSCs-ZSCAN10 $\left(n=5(0 / 5), P^{\star}=6.3 \times 10^{-3}\right)$ and Y-hiPSCs $\left(n=10(0 / 10), P^{\star}<4 \times 10^{-5}\right)$. The number in parentheses represents detected rearrangements and $P$ and $P^{\star}$ are the observed and estimated likelihoods of detecting no rearrangements in the absence of lineage effects using a binomial distribution, respectively ${ }^{50}$. Unprocessed original scans of blots are shown in Supplementary Fig. 7.

Original

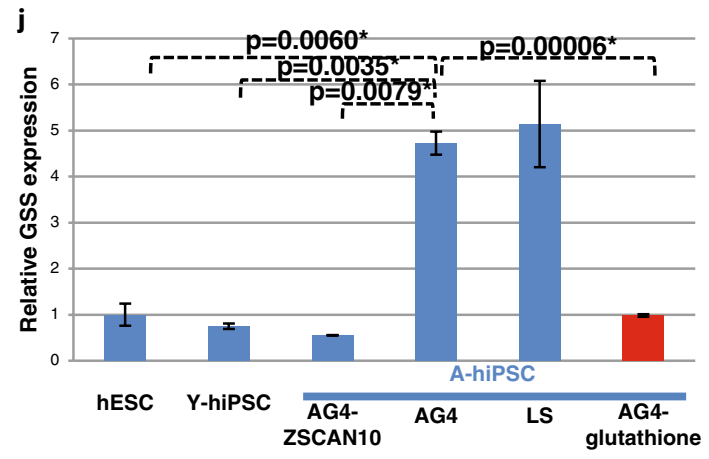

Corrected

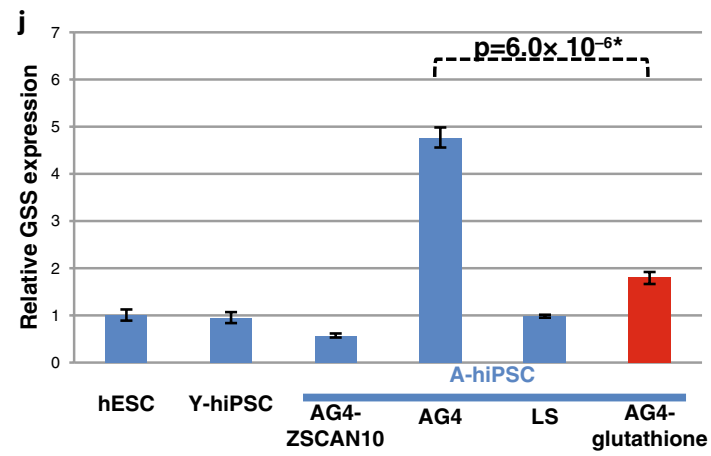

Supplementary Fig. 6 | Higher somatic cell ROS among the tissue donors as a causative origin of the genomic instability in A-iPSC and recovery by glutathione treatment in the early stage of A-iPSC reprogramming. a,b, Somatic cell ROS measured by MitoSOX staining. Mitochondrial Superoxide Indicator, MitoSOX Red dye (ThermoFisher, M36008) was used to measure somatic cell ROS from young somatic cells (Y-SC) from B6CBA mouse and aged somatic cells (A-SC) from B6129 and B6CBA mice (a), and human young somatic cells (Y-SC) from MRC5 donor and human aged somatic cells (A-SC) from LS and AG4 donors (b). Reduced level of the somatic cell ROS with the treatment of the stabilized form of glutathione chemical (3 $\mathrm{mM}$ of glutathione reduced ethyl ester, CAT \#G-275-500, GoldBio) for three days in the media in A-SC from AG4 donor (A-SC-AG4- glutathione) (b). Scale bar, $100 \mu \mathrm{m}$. c,d, Quantification of the MitoSOX staining (ROS) level using image based quantification (ImageJ software) from the samples in Supplementary Fig. 6a,b. Error bars indicate standard error of the mean of independent colonies $(n=10)$. Statistical significance was determined by unpaired two-sided $t$-test. e,f, Representative phenotypes of the reprogrammed iPSC from the donor somatic cells from Supplementary Fig. 6a,b from mouse and human donors. A-SC-AG4 somatic cells with the reduced ROS by the treatment of the glutathione reduced ethyl ester in Supplementary Fig. $6 \mathrm{~b}$, d was studied. $\mathbf{g}$, Immunoblot of pATM showing that A-iPSC with glutathione treatment recover the DNA damage response in the biologically independent clones after phleomycin treatment. A-iPSC were generated with the treatment of $3 \mathrm{mM}$ glutathione reduced ethyl ester prior to and during the early stage of reprogramming (from one day before reprogramming virus infection to 10 days post reprogramming virus infection). $\mathbf{h}$, Copy number profiling analysis of human A-iPSC with glutathione treatment from 10 clones (upper panel). Schematic diagrams represent 10 non-rearranged A-iPSC with glutathione treatment, compared with seven rearranged A-iPSC from 11 clones (lower panel) without glutathione treatment. A-hiPSC ( $n=$ $11(7 / 11), P=0.64$, also presented in Fig. 6e) and A-hiPSC-glutathione $\left(n=10(0 / 10), P^{\star}<4 \times 10^{-5}\right)$. The $P$ values are the observed $(P)$ and estimated likelihoods $\left(P^{\star}\right)$ of detecting no rearrangements in the absence of lineage effects using a binomial distribution, respectively. i, Q-PCR of ZSCAN10. Error bars indicate standard error of the mean of two replicates with two independent clones in each sample group in Supplementary Fig. 6g. Statistical significance was determined by two-sided $t$-test $(n=4)$. Data of the first five columns are also presented in Fig. $5 b$. j, Q-PCR of GSS. Error bars indicate standard error of the mean of two replicates with two independent clones in each sample group in Supplementary Fig. $6 \mathrm{~g}$. Statistical significance was determined by two-sided $t$-test $(n=4)$. Data of the first five columns are also presented in Fig. 5c.

Published online: 14 January 2019

https://doi.org/10.1038/s41556-018-0269-y 